\title{
"Mafias" et politique en URSS
}

\section{Eric Verdier}

\section{(2) OpenEdition \\ Journals}

\section{Édition électronique}

URL : http://journals.openedition.org/conflits/109

DOI : 10.4000/conflits. 109

ISSN : $1777-5345$

Éditeur :

CCLS - Centre d'études sur les conflits lilberté et sécurité, L'Harmattan

\section{Édition imprimée}

Date de publication : 17 octobre 1991

ISSN : 1157-996X

\section{Référence électronique}

Eric Verdier, " "Mafias" et politique en URSS », Cultures \& Conflits [En ligne], 03 | automne 1991, mis en ligne le 31 décembre 2002, consulté le 30 mars 2021. URL : http://journals.openedition.org/conflits/ 109 ; DOI : https://doi.org/10.4000/conflits.109

Ce document a été généré automatiquement le 30 mars 2021.

Creative Commons License 


\title{
"Mafias" et politique en URSS
}

\author{
Eric Verdier
}

L'étude des comportements dits "criminels" est longtemps restée en URSS un domaine tabou. Les programmes de recherche en criminologie, fertiles dans les années qui ont suivi la révolution, se sont vus progressivement privés de moyens à l'apogée des purges staliniennes. En 1936, un auteur soviétique déclarait que le total des crimes commis en URSS en 1935 représentait un peu moins de la moitié du chiffre de 1934. Signe éclatant de la réussite du système, cette criminalité était présentée comme une "survivance de la mentalité capitaliste", en voie de régression. Pendant des années, ce discours du sens commun sera la règle, jusqu'à devenir une croyance communément partagée, mais après la période stalinienne et surtout au sortir de l'ère Brejnev, il va connaître de profonds bouleversements. A la faveur de l'inculpation pour "corruption" de la plupart des alliés de l'ancien secrétaire général, le citoyen soviétique "découvrira" l'existence d'organisations "criminelles" puissantes, véritablement imbriquées dans l'appareil d'État et remettant en cause les fondements même du régime. A la parole rassurante des cadres brejneviens succède le discours pessimiste de la nouvelle équipe dirigeante. Dès 1983, les médias portent sur la place publique les cas "exemplaires" de ces hauts fonctionnaires du parti, autrefois "héros du travail socialiste" devenus brusquement par la simple magie du discours officiel des "mafieux". Par exemple, S. Rachidov, premier secrétaire du parti communiste d'Ouzbekistan, est impliqué (à titre posthume) dans une vaste entreprise de trafic de coton; Tchourbanov, le propre gendre de Brejnev, est condamné pour corruption au terme du procès le plus médiatisé de l'histoire soviétique. La criminalité "organisée", jusqu'alors refoulée et ignorée, émerge alors comme point de convergence de tous les discours politiques. Comment ceux-ci l'instrumentalisent-ils dans les jeux politiques qui deviennent ouvertement concurrentiels? Comment fabriquent-ils l'objet mafia à partir de réalités quotidiennes diverses? On l'aura compris, l'objet de ces quelques pages n'est pas de contribuer à démontrer la "corruption" de la société soviétique d'autant que l'étude des phénomènes dits "criminels" en URSS se heurte à la quasi absence de sources indigènes sérieuses(1). Il s'agit plutôt ici de s'interroger sur les processus sociaux qui font d'anciennes situations de fait, largement acceptées, des "problèmes politiques" pour 
ensuite tenter de jeter les bases d'un cadre théorique d'appréhension de ces réalités multiformes.

Les "mafias" dans le jeu politique soviétique

1-1 La construction sociale d'un problème

a -La symbolique du langage

En 1983, se met en place au Kremlin une nouvelle équipe dirigeante sous l'égide de celui qui était depuis 1967 directeur du KGB, Youri Andropov. Plus qu'une succession logique, l'accession des hommes du KGB au sommet du parti et de l'État se présente comme le résultat victorieux d'une âpre compétition sur le clan des amis de l'ancien secrétaire général, Léonid Brejnev Aussi le premier souci de l'équipe d'Andropov serat-il d'assurer l'élimination politique de cette concurrence encore dangereuse. Dans la logique du KGB, la dénonciation de la "corruption" des anciens dirigeants apparaît comme un moyen légitime pour donner des contours présentables à la recomposition du paysage politique. I1 s'agit même sans doute du seul mode d'action effectivement disponible, si l'on veut éviter un conflit portant directement sur la ligne politique(2). Dès lors, le recours à l'argument mafieux va prendre de l'importance dans les jeux politiques. En 1983 dans l'affaire du trafic de coton en Ouzbékistan, Youri Andropov, profitant de la fermeture du jeu politique, réussit à mobiliser conjointement le KGB, le ministère de l'Intérieur et la procurature (c'est-à-dire les trois principales composantes du système policier) dans la lutte contre les "cadres corrompus". La monopolisation de l'accès au discours public exercée par l'appareil du parti dote l'action gouvernementale d'une totale cohérence. Sur le plan des définitions de situation, il s'agit simplement d'éliminer quelques individus jugés corrompus mais nullement de remettre en cause le système politique et moins encore d'interroger les conditions qui ont rendu possibles de tels actes "criminels".

Seulement en 1987, lors de l'ouverture du procès du gendre de Brejnev, les conditions pratiques de la "lutte" contre la corruption ont changé. En présence d'un champ politique plus ouvert, les dirigeants ont perdu une bonne part du contrôle qu'ils exerçaient auparavant sur les définitions de situations. Dans ce cadre, l'apparition et le succès du terme "mafia" dans l'univers politico-médiatique constitue un véritable événement de langage. En URSS, comme dans la plupart des pays du monde, le mot "Mafia" est assorti d'un capital symbolique puissant. Jusque dans les années soixantedix, il participait des stratégies de stigmatisation du système américain où capitalisme et "criminalité organisée" étaient directement associés. A travers l'usage du vocabulaire se dessine alors l'image sociale d'organisations puissantes et insaisissables, de structures parfaitement hiérarchisées et ordonnancées, tapies dans l'ombre aux côtés des structures officielles de l'État. Les notables corrompus, soudain devenus, par la médiation du discours, des "parrains" n'en acquièrent que plus d'importance et n'en présentent que plus de "dangers" pour le système soviétique. L'action des médias, durant les années qui vont suivre le procès de Tchourbanov, contribuera à élargir le capital symbolique du vocabulaire, et à le doter de nouvelles dimensions en insistant sur l'interpénétration entre mafia et parti à l'échelon local. Associant le terme "mafia" à l'ensemble des activités organisées stigmatisées par le pouvoir, les journalistes vont donc donner du "concret", de la "réalité", à l'idée de la confusion des structures mafieuse et politiques. Dès lors les hommes politiques seront présentés comme les représentants voire les subordonnés d'une seule et même organisation secrète, seule détentrice du pouvoir réel: la mafia du parti. Ainsi a-t-on pu écrire à propos de 
Tchourbanov qu'il n'était sans doute qu'un exécutant dans la hiérarchie de l'organisation de l'ombre. A travers ces discours la mafia existe, la mafia n'est plus contre le parti, elle n'est même plus locale, elle s'identifie au parti. Celui-ci est criminel. Seulement cette identification ne va pas de soi, elle est tributaire des luttes politiques internes sur l'avenir du PCUS et des actions des "agences institutionnelles".

b L'apport d'une caution scientifique

Les premières statistiques sur la criminalité en URSS, publiées en février 1989, sont venues doter le "Problème" mafieux de la caution scientifique des professionnels de la recherche criminologique. On y découvre que la délinquance, et principalement les activités classées dans la rubrique "criminalité organisée" ont fait un formidable bond entre 88 et 89 en dépit de l'énergie considérable déployée pour anéantir les "mafias"(3). Les statistiques criminelles donnent à la menace une réalité à laquelle les journalistes, dépourvus de légitimité scientifique, ne pouvaient accéder. Pour M. Gorbatchev, qui s'exprimait en septembre 89 : "devant les chiffres, l'heure était venue de réagir". Dans les sociétés qui se veulent rationnelles, le recours à ces instruments statistiques qui, quand ils ne sont pas questionnés, ont toutes les apparences de la scientificité, constitue un puissant support de légitimation. I1 faut pourtant souligner que la mesure statistique des écarts aux normes enregistrés par les professionnels du contrôle social se heurte à un considérable problème de méthode. En effet, en dehors même de la question des falsifications de données, de nombreux facteurs vont contribuer à accroître la distance entre ce que les chiffres prétendent montrer et ce qu'ils montrent effectivement. Les lois du silence ou simplement le rapport du citoyen à l'institution policière influent de manière déterminante sur les résultats statistiques. De même, il ne faut pas négliger la portée "des manières de faire" développées par 1'institution policière elle-même, qui sait parfois "fermer les yeux" sur des activités pourtant pénalement sanctionnées. Dans le cas qui nous occupe, on ne peut ignorer la relation entre la construction sociale d'une "menace mafieuse" et l'élévation de la criminalité statistique qui objective cette menace. Dans l'univers des policiers soviétiques, le "problème" prend la forme d'un véritable défi qui remet en cause à la fois les cultures locales et les pratiques routinisées à la base de l'activité quotidienne de contrôle social. La performance, et corrélativement l'importance dans le jeu, des trois principales organisations policières : KGB, procurature, ministère de l'Intérieur, sera mesurée par sa capacité à "juguler la menace", c'est-à-dire concrètement, par sa capacité à arrêter des délinquants quelconques quitte à leur donner de l'importance en justifiant de leur appartenance à une organisation "mafieuse"(4). Ainsi les circonstances pratiques qui ont suivi le décès de Leonid Brejnev ont largement contribué à l'exacerbation de ces concurrences. Le poids du KGB, très sensiblement accru après l'arrivée d'Andropov en 1983 au détriment du ministère de l'Intérieur et de la procurature qui, moins contrôlables, ne bénéficiaient pas de la confiance du pouvoir, est à la base de ces confrontations. L'affaire de la mafia d'Ouzbékistan, qui a vu son apogée lors de l'enquête du juge Gdlian, a longtemps représenté pour les cadres de la procurature un moyen de reprendre l'avantage. Cette course à la "performance", où chaque organisation tente de marquer des points sur les organisations concurrentes, va se traduire par une spécialisation croissante des fonctions policières et la tentation pour chaque organisation d'avoir accès aux ressources étrangères pour gagner la compétition interne. Ainsi, en 1989, le ministère de l'Intérieur crée un département spécial de lutte contre le crime organisé et les pays de la Communauté européenne sont sollicités pour fournir aux policiers soviétiques la technologie et les méthodes dont ils 
ne disposent pas et qui, aujourd'hui, devant le "problème", leur font défaut. Au delà des réformes de structure, l'activité quotidienne du policier soviétique a connu de profonds bouleversements sous l'impulsion de ces nouveaux enjeux. Les classifications indigènes qui lui donnaient les moyens de "fermer les yeux" sur les activités délictueuses jugées bénignes par son organisation se sont heurtées à l'obligation politique de produire des résultats rapides et directement visibles. L'élévation de la criminalité statistique, loin de traduire alors un phénomène social réel, est essentiellement le produit des évolutions de l'appareil policier, désormais doté de plus de moyens pour avoir connaissance des activités pénalement sanctionnées et qui, surtout, voit émerger un intérêt immédiat dans le jeu des concurrences inter-organisationnelles à ne plus couvrir ces activités.

1-2 Les usages politiques du "problème"

On l'aura compris à travers ces dernières remarques, le "problème" mafieux en URSS se construit essentiellement à travers les usages sociaux qui en sont faits. Il serait toutefois abusif de rapporter les usages de la construction sociale de la menace au seul cynisme des acteurs et à des stratégies conscientes. Pour la plupart d'entre eux, aussi bien dans le champ politique que dans l'univers des policiers (gestionnaires professionnalisés de la menace) le problème "a une réalité".

Ainsi, dans le champ politique, on observe un véritable consensus pour reconnaître qu'il existe un problème. Les acteurs se livrent néanmoins un combat sans merci autour de la définition légitime des termes du problème. La position de chacun des acteurs dans le champ de la compétition pour l'accès ou la conservation des postes de pouvoir oriente de manière déterminante ces prises de position. Jusqu'au putsch du 19 août, la "mafia" était conçue par le gouvernement comme un organe étranger au parti mais néanmoins tentaculaire. Le bagage symbolique associé au label permettait de faire l'économie d'une réelle construction du problème tout en offrant aux élites un certain nombre d'opportunités tactiques. La fabrication d'un ennemi unique repérable, phénoménalement semblable aux dissidents "capitalistes" de la période précédente, y fait fonction de bouc émissaire. Ainsi, les déficiences de l'appareil industriel et financier devront être rapportées aux activités "nuisibles" des mafias, assurant ainsi aux cadres du parti la préservation de leur impunité. Le manque de rentabilité et de productivité des entreprises d'État sera facilement expliqué en soulignant l'ampleur des détournements de matières premières destinées à alimenter les marchés clandestins. De même, face au succès mitigé des réformes économiques, comme la loi de 88 sur les entreprises privées, le champ politique trouvera dans la mobilisation du capital symbolique associé aux mafias une explication simple et confortable. L'échec de nombreuses coopératives privées dans le domaine du textile et de la restauration sera ainsi rapporté aux activités des racketteurs et nullement aux difficultés d'approvisionnement liées à la monopolisation étatique des circuits de distribution des matières premières.

En dehors du cadre économique, l'ennemi intérieur va trouver un espace d'instrumentalisation porteur avec l'émergence de revendications "nationalistes" dans les républiques périphériques. Ainsi, de nombreux reportages viendront attester de la complicité entre les "mafias" et les structures indépendantistes, comme par exemple en 1986, lors des émeutes d'Alma Ata au Kazakhstan. La puissance de l'ennemi légitime ainsi les difficultés du gouvernement à faire échec à ces mouvements en même temps qu'elle justifie l'usage de la force à leur encontre. 
Après l'ouverture du champ politique soviétique, les élites au pouvoir vont perdre une large part de leur contrôle sur la définition du problème. Dans le jeu de la compétition pour l'accès aux emplois politiques, des définitions concurrentes se feront jour, avec de surcroît une crédibilité sociale supérieure à celles qui sont énoncées par l'équipe dirigeante. Ainsi, pour Boris Eltsine, dès 1988 la "mafia" et le parti sont une seule et même organisation(5). D'autres acteurs vont fonder l'ensemble de leurs campagnes politiques sur ce thème. C'est par exemple le cas d'une figure "exemplaire" de la lutte contre la "mafia", l'ancien juge d'instruction Telman Gdlian. Nommé pour enquêter sur une affaire de corruption en Ouzbékistan, il va bénéficier de toutes les faveurs du gouvernement en démontrant sur le terrain la "réalité" d'un problème alors parfaitement fonctionnel pour le gouvernement de l'URSS. L'intense mobilisation journalistique autour de la "croisade" de Telman Gdlian va doter son personnage médiatique d'un tel capital d'estime publique qu'il deviendra bientôt pour le pouvoir un piège incontrôlable, usant de son image "d'incorruptibilité", pour accuser les sommets du parti, dont le leader encore très influent des conservateurs, I. Ligatchev. Face à ce "vrai" problème, la procurature n'aura plus véritablement d'autre option que de démettre le juge de ses fonctions, quitte à risquer sa propre image. Stigmatisé pour avoir multiplié les arrestations arbitraires dans un simple souci de promotion personnelle, celui qui, pour la Pravda, était soudain passé en 1989 du statut de héros à celui de "petit juge", se fera élire au Congrès des députés du peuple. On peut prendre toute la mesure de la crédibilité de l'image qu'il voulait donner de lui-même et de l'impact contre-productif de la campagne mise en oeuvre à son encontre quand on sait que $85 \%$ d'électeurs se porteront sur son nom au premier tour parmi 17 candidats.

A travers des indicateurs comme les résultats électoraux, les élites politiques sont en mesure de percevoir le niveau d'objectivation du "problème mafieux" auprès des populations. Bien que faibles d'un point de vue scientifique, ces indicateurs contraignent les tactiques dans le champ politique. L'émergence du "problème" dans le débat politique emprisonne des acteurs qui n'ont d'autre choix que de prendre position et de présenter des solutions, y compris quand leur position dans le jeu a pour conséquence d'affaiblir la crédibilité sociale de leur discours. Ainsi, à la suite de l'affaire Gdlian, le "problème mafieux", opératoire pour les dirigeants en 83 , est devenu un handicap pour l'ensemble des membres du parti y compris son secrétaire général(6). Seulement face à la libéralisation du jeu politico-médiatique, la gestion du handicap représente la seule option viable; il n'est plus possible de nier le "problème". Même aux yeux des putschistes du 19 août, le renforcement de la lutte contre la "criminalité" est apparu comme un mode de légitimation "quasi-naturel". Pourtant dans l'imaginaire des populations, le renversement de Gorbatchev n'était pas loin de s'apparenter à une prise du pouvoir par les "parrains"(7).

II Éléments pour une grille d'analyse de la réalité

Dans l'univers du discours politique, la construction incontrôlée des menaces fonctionne telle une preuve parfaite de la "décadence" du système soviétique. C'est pourtant essentiellement dans ces constructions et dans leurs conséquences sur la réalité qu'il faut voir les ressorts de la recomposition actuelle du régime. En effet, dans l'univers du questionnement sociologique, les phénomènes auxquels renvoie la menace "mafieuse" sont "loin d'être menaçants". A l'inverse, ils correspondent pour la plupart à des catégories construites, relativement balisées, et observables empiriquement à des degrés divers dans toutes les sociétés modernes où, curieusement, elles ne posent pas forcément problème: existence d'un "milieu", utilisation des opportunités 
d'enrichissement offertes par la détention des positions de pouvoir. Sans entrer ici dans les problèmes comparatifs, il conviendra d'établir les bases du cadre théorique indispensables à toute analyse contrôlée de ces phénomènes en URSS.

2-1 Essai de typologie

Le milieu

L'étude des groupes sociaux qui font des activités traditionnellement sanctionnées par l'État, leur profession habituelle, renvoie sans doute à l'aspect le moins "typique" de la "criminalité organisée" en URSS. Sous le label "mafias" on va retrouver en fait l'ensemble des marchés clandestins objets de pénalisation dans les sociétés modernes "rationnelles légales" : trafic de stupéfiants, réseaux de prostitution, racket...

Les criminologues russes se sont parfois penchés sur l'étude des modes d'organisation et des régulations sociales à l'oeuvre dans le "milieu" moscovite. A l'inverse, les informations demeurent beaucoup plus fragmentaires quand il s'agit de s'intéresser à l'ensemble de l'empire soviétique, et particulièrement aux régions rurales. Nous nous limiterons ici à l'examen du premier aspect de la question tout en soulignant l'imperfection du cadre théorique pour l'appréhension globale de ces catégories de marchés. Ainsi, certaines activités clandestines, comme le trafic de stupéfiants, monopolisées par des groupes sociaux professionnalisés dans les zones urbaines, peuvent participer des régulations traditionnelles dans les zones rurales où elles constituent un moteur de l'économie locale(8).

Au premier abord, la structuration de l'univers professionnel des bandits de Moscou ne tient pas tant aux simples opportunités de profits qui s'associent aux activités sanctionnées et dangereuses qu'à des modes de régulation traditionnels largement déconnectés de la réalité du terrain. On voit en effet cohabiter une très forte faculté d'adaptation de la pègre aux opportunités ouvertes par les changements de la société soviétique et des modes d'organisation interne d'apparence archaïsante qui, en raison même de leur aspect décalé, appellent un questionnement. A la veille de la révolution d'octobre, le milieu de Moscou présentait deux caractéristiques pertinentes. D'une part, la "société des bandits" revendiquait une totale séparation du reste de la société. Cette revendication se traduisait dans les faits par un ensemble de règles coutumières sanctionnées par des juridictions internes. Il était par exemple interdit de se marier, d'entretenir une couverture légale ou des relations, de quelque nature qu'elles fussent, avec les représentants de l'autorité d'État. Dans l'univers des bandits, le culte de la marginalité sociale, sans doute issu dans une certaine mesure des "cours des miracles", opérait tel un vecteur de distinction entre les "vrais professionnels" et les "amateurs". La revendication crédible du titre de "vrai professionnel" ou encore de "bandit d'honneur" garantissait au criminel un statut social fait d'estime et d'admiration dans l'espace de ses relations professionnelles. Pourtant, en dépit du rejet de la société, les modes de régulation à l'oeuvre dans la "société des bandits" devaient beaucoup à la structuration traditionnelle de la société russe. Ainsi, le sociologue Valerij Chalidzé(9) a pu retrouver dans les organisations professionnelles du "milieu" de Moscou beaucoup des caractéristiques des corporations de métiers. Tant en matière de désignation des leaders que de répartition des bénéfices ou de sanction des fautifs, les organisations criminelles faisaient rarement preuve d'invention. En des temps où la circulation des personnes était inexistante,

la fermeture de la société des bandits se traduisait par des phénomènes d'autoreproduction. Le fils de voleur n'avait d'autre choix que de devenir voleur lui-même. 
Au XIXème et surtout au XXème siècle, de nouvelles couches de population sont venues concurrencer les "bandits d'honneur" sur leurs propres "marchés". De fait, ils ne représentaient plus en 1917 qu'un très faible pourcentage du "milieu" et la période stalinienne n'a fait qu'accentuer ce mouvement en éliminant dans les camps la plupart des chefs de bandes qui, au nom de la tradition, mais, sans doute, surtout pour préserver le support de leur légitimité professionnelle, refusaient toujours de collaborer avec un pouvoir politique qui les avait pourtant déclarés "socialement proches".

De fait, le milieu moscovite contemporain n'a plus grand chose à voir avec l'univers des bandits du XIXème siècle. Ces dix dernières années ont même connu de profonds bouleversements avec l'apparition de bandes organisées non russes dans la plus connue, "la mafia tchétchène", semble aujourd'hui en position de force sur le marché lucratif du racket à l'encontre des nouveaux coopérateurs. En suivant les opportunités offertes par la société soviétique, les marchés monopolisés par les nouveaux "vrais professionnels" se sont considérablement diversifiés, jusqu'à exploiter, parfois non sans une certaine subtilité, les failles de la législation pénale. Virginie Coulloudon(10) relate par exemple le cas de la vente des véhicules d'occasion où les pratiques induites par l'économie de pénurie conduisent à une forte inflation alors même que les prix officiels sont très encadrés. Sur le grand marché des voitures d'occasion de Moscou, rue Trofimvogo, les transactions n'échappent pas à la vigilance des racketteurs qui prélèvent sur les sommes payées l'ensemble des dessous de table, laissant les vendeurs démunis de tout recours.

Bien que fortement renouvelé, le "milieu" de Moscou voue un véritable culte aux "traditions" des "bandits d'honneurs". Il puise en fait dans un passé très largement "mythifié" les moyens de sa légitimation. Ces instrumentalisations du "passé glorieux" qui ne sont sans doute pas le propre de la pègre soviétique, répondent à de multiples fonctions sociales qui s'expriment dans le présent. Ainsi, on peut voir se nouer entre les bandes rivales des concurrences ?ont l'enjeu semble être la seule reconnaissance du titre valorisé de "bandit d'honneur". Dans la pratique, la reconnaissance du plus ou moins grand prestige d'un gang quelconque par les gangs concurrents repose avant tout sur un rapport de violence. C'est dans le cadre de ce rapport que se réalisent les alliances, que se distribuent les territoires. Pourtant, dans la logique du paraître, ces rapports de violence sont constamment retraduits en terme de prestige. Le "bandit $d$ 'honneur ou encore le "mafieux" est tout simplement celui qui a "voix au chapitre" quand il s'agit de passer un accord. Il se distingue ainsi de "l'amateur" ou du "voyou" qui ne dispose pas de la puissance organisationnelle nécessaire pour accéder aux relations d'échanges avec les autres bandes. Il faut souligner que ces échanges ne se limitent pas à des espaces restreints comme Moscou. Sur certains marchés comme le trafic de stupéfiants, ils peuvent se réaliser à l'échelle de l'URSS. Certains observateurs relatent la tenue de véritables congrès où se jouent, dans une atmosphère de violence aseptisée, les répartitions de territoires(11).

La valeur de la quête de prestige des "bandits d'honneur" n'est toutefois pas simplement instrumentale. A travers l'image mystifiée du "vrai professionnel", la pègre russe joue sa propre image. Le mythe de Robin des bois, associé au "milieu" du XIXème siècle dans la culture romanesque des Moscovites donne aux "mafias" modernes l'assurance d'un regard relativement bienveillant de la part des populations. Ainsi, les 
rackets organisés à l'encontre des nouveaux millionnaires issus de la loi de 88 sur les entreprises privées ne soulèvent-ils pas de vives protestations.

On peut apercevoir la réalité des "traditions" du milieu et des rapports sociaux attachés à ces "traditions" quand ceux-ci perdent leur fonctionnalité. Ainsi, la marginalité, symbole de statut social avant la révolution, est devenue aujourd'hui un insurmontable handicap. Le "mafieux" ne peut espérer prospérer dans le "milieu" sans passer des compromis avec la société et en particulier avec les autorités chargées de mettre fin à ses activités.

Les entrepreneurs clandestins

A travers la catégorie "entrepreneurs clandestins" nous désignerons un phénomène sensiblement décalé de celui examiné plus haut et sans doute beaucoup plus caractéristique des économies soviétisées. L'entreprise clandestine se présente comme la conséquence structurelle de la rencontre entre la pénalisation de l'industrie privée et les déficiences du système de production d'État. Deux points d'inégale valeur permettent de distinguer l'entreprise clandestine des activités monopolisées par le "milieu". En premier lieu, sont visés ici des marchés portant sur des biens de consommation licites. La pénalisation tient simplement dans l'enrichissement "antisocialiste" de personnes privées. Cette remarque serait sans conséquence si elle ne débouchait sur la seconde caractéristique de la catégorie. Dans l'univers de la construction sociale de la réalité les entrepreneurs clandestins sont étrangers au "milieu". Pour les "vrais professionnels", ils représentent moins des confrères ou des concurrents que des "clients". On observe ainsi que les marchés pris en charge par les organisations tendent à fonctionner comme des vecteurs de classement tacite dont dépend la nature des rapports entre ces organisations. Pour le "vrai professionnel", pour celui qui, en tout cas, se définit comme tel avec un minimum de crédibilité sociale, l'entrepreneur clandestin passe pour un "honnête homme", intégré à la société au même titre que les autres citoyens. I1 ne fera pas l'objet d'un traitement différent. On ne connaît en fait aujourd'hui que fort peu de choses des entreprises clandestines, sinon à travers les cas anecdotiques rapportés par la presse soviétique. Il semble néanmoins que l'ampleur du phénomène soit considérable bien qu'il ne faille pas prêter trop d'attention aux tentatives de chiffrage. Les auteurs s'accordent pour situer le démarrage des économies clandestines "industrielles" dans les années cinquante, voyant généralement dans la répression stalinienne un sérieux obstacle à leur développement. Cette explication souple pointe le doigt sur le relâchement du contrôle politique qui a suivi la mort de Staline et sur les nouvelles opportunités offertes par la remise en cause partielle de l'idéologie officielle. En effet, si le "milieu" était considéré comme nuisible, le discours politique stalinien le présentait néanmoins comme "socialement proche". A l'inverse, la logique de l'entreprise privée heurtait de front les fondements du dogme. Bien que commode, cette grille d'analyse de la réalité n'est que partiellement satisfaisante. Elle présuppose en effet une totale continuité entre le discours et les règles du jeu effectives du système, continuité dont la preuve est loin d'être établie. Le développement d'une économie parallèle dans un système soviétisé gagne à être rapporté à la structure même de ce système. On sait par exemple que la monopolisation des activités industrielles par les entreprises dépendantes de l'État prive les entrepreneurs privés de tout moyen d'approvisionnement licite. De fait, l'émergence d'une économie privée autre qu'artisanale est étroitement dépendante du rapport des entrepreneurs clandestins à l'industrie officielle. Ce n'est sans doute pas un hasard si les premiers ateliers privés "découverts" au sortir de la guerre dans le 
domaine de la confection l'ont été dans des zones fortement industrialisées où la rareté des matières premières n'était que relative(12). En droit soviétique, le "vol de biens socialistes" est considéré comme l'un des crimes les plus graves. Cette classification dogmatique est loin de correspondre aux valeurs partagées par la population. Quand il est réalisé sur une petite échelle, le vol dans les entreprises d'État apparaît comme un acte normal. En dehors même de la satisfaction des besoins personnels il peut parfois se présenter sous le jour d'une compétition dans laquelle les joueurs jouent leur image autant qu'ils spéculent.

En ouvrant de larges parts de l'économie aux entrepreneurs privés, la loi de 88 sur les "coopératives" n'a pas pour autant résolu le "problème" de l'industrie clandestine. Dans le domaine du textile d'habillement par exemple, de nombreux obstacles freinent la conversion des structures existantes, spécialement adaptées au système de clandestinité et aussi peu aptes à se réformer que ne le sont les entreprises d'État. Au delà de la question des impositions fiscales, souvent mises en avant, l'entrée dans l'univers de la légalité remet en cause tant les méthodes d'approvisionnement que les moyens de production ou les circuits de distribution. Ainsi, la tenue d'une comptabilité exclut le recours au vol de matières premières alors même qu'il n'existe aucune autre méthode effectivement disponible pour se les procurer. Le danger, réel ou fictif, lié à la clandestinité, en justifiant la pratique de bénéfices exorbitants, est le seul garant de la rentabilité financière de ces organisations qui, de fait, ne sont pas fondamentalement plus productives que les entreprises d'État mais jouissent néanmoins d'un quasimonopole face à l'industrie privée légale freinée dans son développement par les pesanteurs administratives.

Les usages sociaux des positions de pouvoir

Ce troisième aspect des "criminalités organisées" en URSS est sans doute le mieux balisé d'un point de vue théorique dans la mesure où il renvoie à des problèmes observables dans l'ensemble des sociétés modernes. L'une des propriétés des organisations bureaucratiques tient dans leur faculté à développer des manières de faire à définir des catégories d'enjeux qui échappent au champ de leurs compétences légitimes strictement encadrées par 1 ordre juridique. Ces manières de faire, reconnues comme légitimes par les membres des organisations, ou pour le moins incontournables, restent néanmoins le plus souvent inavouables. Ce sera par exemple le cas de ces activités incriminées sous le label "corruption" qui constituent parfois dans la logique interne des organisations, fruit de leur parcours historique, des modes de régulation normaux et acceptés.

L'affaire des détournements de coton en Ouzbekistan, qui a fait la "une" des journaux soviétiques durant plusieurs années et au terme de laquelle la plupart des cadres du ministère de la production cotonnière a été limogé, nous livre quelques clés pour la compréhension de ces phénomènes. Dans l'univers traditionnel des sociétés musulmanes d'Asie centrale, certaines manières de faire, aujourd'hui stigmatisées, participaient de l'économie régulière du système de gouvernement. Ainsi en était-il de la pratique des cadeaux qui consistait à offrir ses biens les plus rares aux personnes dont on pouvait légitimement attendre un service. Loin de se présenter sous le jour d'un échange, le système des cadeaux misait sur l'inattention à la réciprocité immédiate. Il permettait simplement la constitution d'un capital d'alliés influents dont on attendait qu'ils fussent mobilisables en cas de besoin. La confiance liée à 
l'enracinement social de ces rapports garantissait leur pérennisation. C'est ce que l'on a appelé "le clientélisme".

On ne peut nier les déterminismes historiques que font peser ces pratiques traditionnelles sur les modes de régulation sociale à l'oeuvre dans les grandes bureaucraties constituées à la suite de la révolution d'octobre. Toutefois, l'adaptation des manières de faire aux structures soviétisées ne se réalise pas sur un mode linéaire. A travers ces pratiques, vont se nouer des enjeux spécifiques aux organisations, vont se constituer des règles du jeu. Ainsi, pour le fonctionnaire du ministère de la production cotonnière, la détention d'un pouvoir de sanction va rapidement apparaître comme une ressource facilement monnayable. En fermant les yeux sur certaines activités, ou en ne cherchant pas à les connaître, il s'assurera l'essentiel de sa rétribution. Corrélativement, la valeur d'une position à l'intérieur de l'organisation ne s'appréciera plus tant au regard de son prestige officiel qu'en fonction des potentialités de rétribution occultes qu'elle offre à son détenteur. De ce fait, les emplois publics rentables deviendront eux-mêmes des biens monnayables, des valeurs de placement plus que des emplois effectifs. Durant la période brejnevienne, l'enracinement de ces règles du jeu dans la plupart des secteurs de l'État va garantir leur solidité. En bénéficiant des cadeaux des responsables locaux, le secrétaire général saura fermer les yeux sur les cadeaux qu'ils reçoivent eux-mêmes. Certains hauts responsables, comme Tchourbanov, investiront l'essentiel de leur temps dans la recherche de ces gratifications.

Le propre des règles pragmatiques du jeu, quel qu'en soit la nature, est d'exercer un effet d'autocontrainte sur les secteurs sociaux dans lesquels elles émergent. Les acteurs n'ont le plus souvent d'autre choix que de jouer le jeu quand bien-même ils n'approuvent pas forcément les termes. L'affaire du coton d'Ouzbékistan recèle un certain nombre d'exemples d'assassinats de responsables "honnêtes". Pourtant, la logique du système s'oppose à ce que l'élimination constitue un mode de régulation normal du jeu. En effet, l'accès aux positions de responsabilité est étroitement dépendant de l'acceptation, au moins instrumentale des règles. On peut ainsi difficilement imaginer que le président Gorbatchev se soit exonéré des manières de faire reconnues dans le jeu politique précédent pour accéder aux fonctions suprêmes. L'ouverture du champ politique remet fondamentalement en cause les règles de la compétition pour l'accès aux postes de pouvoir en même temps qu'elle influe sur la définition des enjeux et des priorités pour une large gamme d'acteurs. Dans l'univers des journalistes, par exemple, la dénonciation participe de l'image que les organisations veulent donner d'elles-mêmes. La concurrence entre les entreprises de presse autour de la fabrication crédible d'une image d'indépendance va générer de véritables scandales politiques, processus de mobilisation au parcours chaotique et incontrôlé, d'autant plus problématiques pour les anciens dirigeants qu'ils constituent, du fait même de la forte demande des journalistes en ce sens, une ressource immédiatement disponible pour les nouveaux entrepreneurs politiques dans le champ de leur compétition propre quand il s'agit de dénoncer un concurrent comme "mafieux". La nouvelle structure du combat politique, finalement assez proche de celle que l'on observe dans les démocraties occidentales, contraint les acteurs non seulement à se donner une image présentable mais encore, et surtout, à "mieux choisir leurs amis", c'est-à-dire à éviter effectivement toutes les "compromissions" qui, compte tenu des propriétés du jeu, sont susceptibles de "faire scandale".

2-2 Le "système mafieux" en URSS 
- Une logique de jeux transversaux

Dans l'univers du discours, la confusion des phénomènes, largement incontrôlée, contribue à entretenir l'image d'organisations mystérieuses, cristallisations de toutes les peurs sociales. Derrière la construction politique on a sans doute affaire à une réalité banale, qui peut en tout cas être rapportée à des logiques observables dans l'ensemble des sociétés modernes. Le concept d'organisation, traduction de réalités très diverses, se révèle le plus souvent imparfait pour appréhender les rapports sociaux à la base des "criminalités organisées" dans toute leur complexité et la réflexion gagne en intelligibilité quand on lui substitue d'autres grilles d'analyse. En effet, la puissance, ou plus exactement la solidité, d'un système quelconque ne dépend pas tant des effectifs respectifs de chacune des organisations qui le composent que de la nature des rapports qu'elles entretiennent entre elles. Observer ce que l'on pourrait appeler, par économie de langage, le "système mafieux" conduit à s'intéresser avant toute chose à l'ensemble des jeux transversaux observables entre les divers univers sociaux dont nous avons dressé les contours.

C'est sans doute dans le domaine des rapports entre le secteur politique et les entrepreneurs clandestins que l'on peut parler avec le plus de certitude de système consolidé dans la mesure où il s'a $t$ ici dune véritable logique d'interdépendance. Pour l'entrepreneur clandestin, dont l'activité est de fait dotée d'une forte visibilité sociale, l'entretien de relations avec les autorités chargées de le sanctionner est une condition de la survie. Le jeu transversal consolidé bouleverse les manières de faire des organisations qui le composent. Ainsi, pour le responsable du parti ou le policier il n'existe plus de véritable choix possible entre l'acceptation des pots de vin et l'exercice "normal" de ses compétences. Loin de se présenter comme des opportunités à saisir, les sollicitations extérieures s'imposent sur le mode du "cela va de soi". Par exemple, en Ouzbékistan, le remplacement d'une large majorité des cadres semble être resté sans effet sur le système des jeux transversaux. En 1983, le nouveau premier secrétaire de la république, Inamjon Ousmankhodjaev fustigeait à la tribune du Congrès à Moscou les pratiques de corruption de son administration. En 1988, il "tombait" pourtant lui-même sous ce chef d'inculpation. Tout comme les autres, il avait dû jouer le jeu faute de pouvoir le transformer. L'existence d'un système de transactions consolidé entre les entreprises clandestines et les administrations de l'État est sans nul doute l'une des causes directes de l'échec des lois sur l'entreprise privée et de la persistance des organisations parallèles. En effet les cadres de l'État ont tout autant à perdre que les entreprises clandestines dans ces réformes qui, à travers la légalisation de l'appropriation privée des moyens de production, remettent en cause leurs principales ressources coercitives et corrélativement leur valeur marchande dans le jeu de la corruption.

Face aux systèmes de rapports consolidés, la position du "milieu" semble sensiblement décalée dans la mesure où rien ne permet de parler d'interdépendance. Selon Virginie Coulloudon, qui s'inspire des sources officielles disponibles, le racket des entreprises clandestines représente pour les "vrais professionnels" un chiffre d'affaires de loin supérieur à celui du trafic de stupéfiants ou de la prostitution. Plus qu'un système de transactions, c'est essentiellement la violence qui caractérise les rapports entre ces deux univers sociaux, ce qui n'exclut pas l'existence d'alliances personnalisées, comme par exemple en matière de "contrats" pour l'élimination de concurrences gênantes. Des 
exemples historiques attestent en revanche de l'existence de collaborations solides entre certaines sociétés de bandits et le champ politique. Avant la révolution d'octobre, alors que le parti Bolchevik était encore étranger à l'espace politique légitime, des collaborations ponctuelles ont parfois permis de "remplir les caisses". Durant les années de guerre civile, les "socialement proches" étaient souvent recrutés en vue de participer aux "expropriations". Les autorités soviétiques ont toujours tenté de minimiser ces collaborations rendues illégitimes par la "suite de l'histoire". Pourtant, elles étaient en 1918 reconnues par la loi qui allait jusqu'à fixer des barèmes de rétribution. C'est plutôt sur le terrain des rapports personnalisés qu'il faut examiner aujourd'hui les transactions entre les "vrais professionnels" et le champ politique. En effet, il n'existe pas, à l'opposé de l'entreprise clandestine, de rapport d'interdépendance structurel entre ces deux univers sociaux et seule la corruption interne de l'appareil policier est en mesure de préserver la solidité des transactions existantes, sauf à postuler du multi-positionnement des acteurs.

Vers un hypothétique multipositionnement des acteurs

Le cadre théorique dressé ici repose sur l'isolement de secteurs d'activité particuliers et l'observation des échanges entres ces secteurs. Certains phénomènes, dont on ne peut encore mesurer la portée, faute de données empiriques contrôlables, viennent toutefois brouiller la grille d'analyse.

Jusqu'au début des années 60 , le jeu politique soviétique présentait un aspect relativement fermé. L'accès aux écoles du parti, qui ouvraient la porte aux postes de pouvoir, supposait des tests de sélection sévères afin de s'assurer de la fiabilité des postulants. De fait, les familles déjà implantées dans le secteur bénéficiaient d'un avantage certain. Cette logique d'auto-reproduction contrôlée va contribuer à la pérennisation de règles du jeu parfaitement codifiées et, corrélativement, de manières de faire aisément anticipables. Les pots de vin, appréhendés dans la logique du jeu comme des modes de rétribution légitimes, vont contribuer à solidifier le système en raison de l'accès privilégié des membres du parti aux ressources monétaires de l'État. L'émergence d'une économie clandestine quasi-industrielle va progressivement amener la rupture de ce monopole, donnant aux millionnaires occultes les moyens d'entrer dans le jeu. Pour l'entrepreneur clandestin et peut-être aussi certains membres du "milieu", la possession du capital monétaire va devenir le meilleur passeport pour l'accès aux positions de pouvoir dans le parti et les entreprises d'État. Ainsi il était semble-t-il possible au début des années soixante-dix d'acheter un poste de secrétaire de district pour environ 200000 roubles (le salaire moyen était alors de 1500 roubles). Dans les républiques d'Asie centrale on a vu apparaître à cette époque de nouveaux responsables cumulant les activités politiques, la fabrication clandestine de biens de consommation et le contrôle du trafic des stupéfiants. Ainsi en était-il par exemple d'un dénommé Adylov, officiellement directeur d'un trust industriel aujourd'hui en prison. Ce phénomène de multipositionnement, s'il est confirmé empiriquement à une plus grand échelle, appelle de nouveaux questionnements; on ne peut en effet négliger les incidences des cumuls de rôles sur l'autonomie des jeux sociaux dans lesquels s'insèrent ces rôles. La multiplication des catégories d'enjeux, la coexistence de manières de faire issues de logiques sociales distinctes dans un même jeu aux frontières difficilement perceptibles, réduit les facultés de calcul et d'anticipation des acteurs positionnés dans le jeu. L'éclatement et la désorganisation des règles pragmatiques à l'oeuvre dans le parti communiste d'URSS est peut- être aussi l'un des ressorts structurels d'une large gamme de rapports conflictuels, qu'ils 
prennent le nom de "lutte contre les mafias" ou bien, parfois, de "chasse aux conservateurs", de "chasse aux communistes".

\section{RÉSUMÉS}

La criminalité est longtemps restée en URSS un sujet tabou. Elle constitue pourtant aujourd'hui le point focal de tous les discours politiques. L'auteur s'attache à l'observation des usages sociaux que les acteurs dénomment mafieux pour en montrer la diversité : criminalité traditionnelle, entrepreneurs clandestins, enrichissement par le biais de position politique. Il propose de comprendre le développement du discours sur les mafias en parallèle avec l'ouverture du jeu politique et la perte de monopole du parti communiste. L'accusation mafieuse ne renvoie donc pas à un phénomène homogène même si certains acteurs sont multipositionnés, en revanche c'est un ressort efficace dans le nouveau jeu politique pour les principaux leaders et aussi pour les agences institutionnelles : média, police, justice.

Criminality has been a taboo subject in the USSR for a long time. Today, it constitutes the common denominator of all political speeches. Through the observation of social customs, called Mafia by the actors, the author shows its diversity : traditional criminality, illicit contractors, enrichment through political positions. His suggestion to the understanding of the development of political references to Mafia is that of putting it into parallel with the opening of the political game and the communist party's monopoly loss. The accusation of belonging to Mafia does not, therefore, refer to an homogeneous phenomenon even if some actors are " multipositioned ". Nevertheless, it is an effective force in the new political game for the main leaders as for institutional agencies : media, police, justice.

\section{INDEX}

Index géographique : Russie

Mots-clés : mafias et crime organisé, contrebande et trafic

\section{AUTEUR}

\section{ERIC VERDIER}

Doctorant en science politique à l'Université Paris-X-Nanterre 